Itinéraires Itinéraires

Littérature, textes, cultures

\title{
L'ethos collectif des professeurs documentalistes sur Twitter : exploration de quelques pratiques
}

Collective Ethos of School Librarian Teachers on Twitter: Exploration of Some Practices

Florence Thiault

\section{OpenEdition}

\section{Journals}

Édition électronique

URL : http://journals.openedition.org/itineraires/3138

DOI : 10.4000/itineraires.3138

ISSN : 2427-920X

\section{Éditeur}

Pléiade

\section{Référence électronique}

Florence Thiault, «L'ethos collectif des professeurs documentalistes sur Twitter : exploration de quelques pratiques », Itinéraires [En ligne], 2015-3 | 2016, mis en ligne le 01 juillet 2016, consulté le 01 mai 2019. URL : http://journals.openedition.org/itineraires/3138; DOI : 10.4000/itineraires.3138

Ce document a été généré automatiquement le 1 mai 2019.

\section{$\Theta \Theta \Theta \Theta$}

Itinéraires est mis à disposition selon les termes de la licence Creative Commons Attribution - Pas d'Utilisation Commerciale - Pas de Modification 4.0 International. 


\section{L'ethos collectif des professeurs documentalistes sur Twitter: exploration de quelques pratiques}

Collective Ethos of School Librarian Teachers on Twitter: Exploration of Some Practices

Florence Thiault

1 Nous souhaitons interroger les formes de visibilité en ligne sur Twitter des professeurs documentalistes en charge des centres de documentation et d'information (CDI) dans les établissements scolaires du second degré. À partir d'une analyse des stratégies d'acteurs (gestion de leur image, formes de présence, productions numériques, interactions followers-followings, etc.), nous étudions le lien entre la présentation de soi sur Twitter et la définition d'une identité professionnelle collective. Une enquête du site Savoirs $C d i^{1}$ nous informe sur l'adoption du réseau Twitter dans cette profession parmi les autres réseaux sociaux numériques (RSN) disponibles. Il apparaît comme le réseau social le plus utilisé à des fins professionnelles (34\% des réponses des enquêtés). La logique d'usage repérée consiste principalement à réaliser une veille informationnelle sur des thématiques choisies, à diffuser des informations et à partager des contenus autour de centres d'intérêt entre pairs. L'objectif pour le professeur documentaliste est de favoriser le processus de mise à disposition, de traitement, d'accès et d'appropriation de l'information pour les usagers, et de façon élargie pour la communauté professionnelle. C'est une démarche qui vise à mettre en œuvre des dispositifs info-communicationnels à des fins de diffusion des savoirs (Thiault 2016). Ce site de micro-blogging est le lieu de stratégies de la part des individus qui l'utilisent pour se rendre visibles aussi bien socialement que professionnellement. La construction discursive d'une image de soi participe à l'élaboration d'un «ethos » au sens de Max Weber (1996), c'est-à-dire d'un ensemble de valeurs partagées orientant des pratiques professionnelles. 
2 Pour dégager un "ethos professionnel», révélateur des facettes de l'identité professionnelle des professeurs documentalistes, nous nous appuierons sur le modèle sémiotique de l'identité numérique défini par Fanny Georges (2008). Cette dernière distingue trois ensembles de signes : l'identité déclarative, agissante et calculée. L'identité déclarative se compose de données saisies par l'utilisateur (nom, fonction, photographie, centres d'intérêt, etc.). L'identité agissante se caractérise par le relevé des traces d'activité de l'utilisateur (navigation, demande d'amis, téléchargements, etc.). Quant à l'identité calculée, elle se manifeste par les calculs effectués par le système logiciel (nombre d'amis par exemple). Le concept de dispositif techno-sémiopragmatique développé par Daniel Peraya (1999) nous éclairera sur l'influence du dispositif pour l'analyse des formes de l'identité agissante et calculée. Dans cette étude, nous examinerons l'identité déclarative en tant que processus de construction et représentation de soi en ligne, et l'identité agissante et calculée comme développement de signes d'audience et de notoriété. Après avoir présenté nos modalités d'exploration des stratégies de présence en ligne des professeurs documentalistes, nous proposerons une distinction entre différentes formes d'ethos professionnels identifiés dans notre corpus. La dernière partie portera sur les formes d'audience dans la communauté professionnelle étudiée.

\section{Stratégies énonciatives de présence en ligne}

3 Twitter est particulièrement adapté comme outil d'échanges entre pairs et support de présentation de soi dans une visée de définition d'une identité professionnelle. La visibilité de la présence (des actions, des échanges) est devenue un enjeu professionnel. Sur et via Twitter, elle participe d'une légitimation du groupe professionnel des professeurs documentalistes. Cette dimension interroge la question de l'auctorialité des productions numériques sur Twitter et celle de l'e-réputation d'experts dans un champ professionnel spécifique. Une pratique récurrente chez les utilisateurs consiste à insérer des liens dans les messages vers des pages extérieures, l'autorité dans ce contexte a pour effet d'informer (Broudoux 2007). Nous ne pouvons considérer Twitter comme un espace autonome sans prendre en compte la complexité des pratiques de sociabilité en ligne dont la dimension constitutive est la dissémination de l'information. Ce RSN constitue un des éléments d'une stratégie de présence numérique liée à d'autres dispositifs (blog, portail du CDI, page Facebook, compte Netvibes, etc.). En effet, le développement des réseaux socionumériques suscite une nouvelle forme d'accès aux ressources informationnelles sous forme de prescriptions et de recommandations (Coutant et Stenger 2010).

4 Afin d'analyser les démarches et stratégies en jeu dans ce groupe d'acteurs, nous nous fondons sur le cadre de l'anthropologie de la communication pour l'observation de traces d'interactions qui constituent des marqueurs d'une culture professionnelle. Nous nous appuyons sur les signes iconiques et linguistiques, en mobilisant la sémiotique pour observer différentes modalités de communication organisées et affichées par les profils Twitter d'un corpus restreint. L'ensemble des ressources Internet incorporées au profil participe de l'identité numérique du sujet. Cette dernière est constituée d'un ensemble de données personnelles, un construit langagier et discursif qui compose des traces investigables. L'hexis numérique est une représentation vécue et expérimentée en contexte interpersonnel dans les dispositifs en ligne ou en réseau. Fanny Georges (2008: 
1) la définit comme "une sculpture agissante de soi dans le monde virtuel». Cette représentation de soi se manifeste par des signes observables à l'écran. L'identité numérique se tisse entre l'ensemble des signes saisis par le sujet et l'ensemble des signes valorisés par le dispositif. Cette manifestation de l'emprise culturelle de la communication en ligne est « dépendante à la fois de la structuration de l'identité propre au dispositif et à la fois dépendante de son actualisation par la communauté des utilisateurs dont fait partie le Sujet (la "culture locale") » (Georges 2009 : 78).

Notre étude de nature empirique se fonde sur une approche microsociale d'une population particulière composée de professeurs documentalistes investis sur la plateforme numérique de réseau social Twitter. Notre objectif central est d'identifier les stratégies des acteurs en ce qui concerne la gestion de leur image et de leurs productions par l'étude des formes de présence et de visibilité. Nous avons délimité notre corpus à partir des données d'un annuaire collaboratif et évolutif de comptes de professeurs documentalistes et de CDI mis en œuvre par une formatrice sur un site dédié (http:// twittezvous.fr/). L'inscription dans l'annuaire (créé en 2011) est volontaire et nous permet ainsi d'interroger des comptes identifiés qui présentent cette affiliation professionnelle. Après exploration, nous retenons 113 profils individuels actifs ${ }^{2}$ à analyser. Nous avons choisi d'exclure les comptes collectifs et les profils non publics (qui impliquent un niveau de confidentialité pour certaines données), ainsi que ceux d'enseignants-chercheurs et de formateurs afin d'observer prioritairement des professeurs documentalistes en exercice en établissement scolaire.

\section{Présentation de soi et identité professionnelle}

6 Sur Twitter l'identité affichée par les professeurs documentalistes est tout à la fois professionnalisée et personnalisée. Valérie Jeanne-Perrier insiste sur le rôle crucial du profil :

Il s'agit d'occuper une place professionnelle en la faisant advenir dans l'acte de production d'une matière médiatique et de sa mise en circulation, en s'appuyant sur un travail éditorial et discursif, autour du «profil», forme standard et rhétorique située aux fondements des règles d'usages du dispositif. (Jeanne-Perrier $2013: 264)$

7 L'investissement dans la création des profils apparait considérable, cette mise en scène de soi nécessite une attention continue et un travail de création d'une «bulle » personnelle, c'est-à-dire d'un espace propre, d'une identité. «Les bulles sont aménagées, décorées et constamment mises à jour " (Rieder $2010: 17)$. L'identité déclarative est composée du couple pseudo/avatar (Georges 2009) fondé sur des marques indicielles de la personnalité du sujet et des éléments d'un discours d'appartenance à un collectif. La présence d'une biographie offre la possibilité d'identifier le statut que l'internaute se donne (de nombreux profils observés se décrivent de manière attendue comme professeur documentaliste), ainsi que les thématiques qu'ils souhaitent aborder sur leurs comptes.

\section{Le pseudonyme et l'avatar}

8 L'internaute est présent dans l'échange en ligne et peut interagir avec les autres par l'entremise d'un personnage qui est son représentant à l'écran. Se nommer est un acte qui permet au sujet social de s'inscrire dans un groupe d'appartenance. Les pseudonymes 
sont sémantiquement évocateurs, et porteurs d'un fort individualisme expressif (Allard 2007), une "technique du soi» au sens de Foucault (1988). La particularité de la dénomination en ligne réside dans le fait que les noms à l'écran ont été choisis et non imposés, comme les identités civiles. Ces stratégies communicationnelles correspondent à des aspirations individuelles à exprimer un «soi " profond. Ce mode de dénomination autonome est un des éléments à travers lesquels la présence en ligne des usagers peut être étudiée. Certains internautes capitalisent leur e-réputation sur leur vrai nom, donnant une visibilité à leurs actions. En effet, la majorité des utilisateurs de notre corpus choisissent un nom qui intègre une référence à leur patronyme et à leur prénom. L'usage du «je» par le sujet traduit l'importance du moi dans le discours. L'utilisation des fragments de noms en lien avec la sémantique du nom propre participe également de l'ethos discursif du sujet. Une difficulté liée au dispositif technique de Twitter est de trouver un nom libre; ce problème oblige le nouvel entrant à associer éléments langagiers et chiffres pour se distinguer. Quant au rattachement à une identité collective, il est marqué par le choix de pseudonymes qui intègrent dans leurs sémantiques le nom $\mathrm{du} \mathrm{CDI}$ où exerce le professeur documentaliste. La fonction professionnelle est également mise en avant dans les noms qui utilisent le même suffixe de production «doc». L'élaboration du sens sur le Web est donc collective et partagée à travers cette appellation générique de « doc » pour « documentaliste ».

Fig. 1. Pseudonymes et ethos professionnel ${ }^{3}$

\begin{tabular}{|c|c|}
\hline Pseudonymes avec « CDI » & Pseudonymes avec « doc » \\
\hline cdideGaulle & Doc_en_stock \\
\hline virtoolcdi & Doc_And_Cie \\
\hline MartheAuCDI & Docabord \\
\hline obiwancdi & Sofydoc \\
\hline cdiblandine & ProfDocDoc \\
\hline Cdidoc31 & viededoc \\
\hline cdischuman & AWebdoc \\
\hline
\end{tabular}

9 Ainsi, il existe un certain alignement des pseudonymes sur les contraintes identitaires de la communauté thématique, la sémanticité s'avérant contextuellement située. Les traces de présence illustrent des traits et des références culturelles des personnes qui y ont recours. En choisissant un nom d'animal ou de personnage célèbre, les internautes peuvent ainsi suggérer qu'ils partagent quelques qualités avec ces derniers.

10 L'association du pseudonyme et de l'avatar sur Twitter nous montre que le pseudonyme est également dépendant de l'ensemble des données numériques du porteur. L'avatar accentue le sens du " pseudo » et inversement. Les pseudonymes sont aussi des masques discursifs qui offrent la possibilité de s'exprimer librement en assurant une sécurité communicationnelle en ligne. 
Fig. 2. Relation entre l'avatar et le pseudonyme

\begin{tabular}{|r|c|}
\hline Pseudonyme & Avatar \\
\hline Herisson08livre & \\
\hline Vache'mentdoc & \\
\hline minoudoc & CMagali Martin \\
\hline
\end{tabular}

11 Sur Twitter, le personnage avatar correspond le plus souvent à une photographie type portrait d'identité, une image naturaliste où la personne est potentiellement reconnaissable. Les portraits d'utilisateurs affichant leur visage (49 profils) se mélangent aux photographies retouchées (11 modifications de paramètres de forme, couleur et texture). Une pratique courante dans les RSN consiste à choisir un personnage numérique, une image idéalisée de soi. «Une incarnation faite de pixels, derrière laquelle un acteur social cherche à exprimer ses exigences, ses goûts, ses dispositions " (Casilli 2012 : 16). La présence en ligne passe également par une mise en scène du corps, par l'association avec une figure synecdoctique. Quelques profils (8) représentent ainsi une partie symbolique du corps (ombre, pas, pieds, œil, jambes, etc.). Quant aux avatars anthropomorphiques, ils mettent en œuvre un processus d'identification et de reconnaissance de représentations visuelles d'animaux familiers comme le chat (12 profils). Les avatars se posent comme les prolongements visuels et graphiques du pseudonyme, renforçant la signification que donnent les acteurs à leur profil.

\section{Images de l'ethos professionnel}

L'ethos professionnel est constitué par les «dispositions acquises, par expérience et relatives à ce qui vaut plus ou moins sur toute dimension (épistémique, esthétique, sociale, etc.) pertinente dans l'exercice d'un métier» (Zarca 2009 : 352). Il constitue une source d'identification et de reconnaissance pour les membres d'un groupe professionnel, ce qui n'empêche pas la stratification et la segmentation de celui-ci. Nous pouvons faire correspondre plusieurs des registres mis en évidence lors de l'analyse de l'identité déclarative de notre échantillon à des ethos spécifiques. De la sorte, nous distinguons 
différents ethos professionnels adoptés par les professeurs documentalistes. Ces ethos professionnels ont des caractères communs avec ceux de professions semblables.

\section{Ethos de professionnel du livre} avec le métier de bibliothécaire est courante. Plusieurs avatars (19) représentent des lecteurs, des livres, des étagères de bibliothèques, ou des plumes pour écrire à l'encre qui renvoient à la figure de l'écrivain. Par ailleurs, une des missions du professeur documentaliste auprès des élèves est de favoriser l'accès à la lecture. Les personnages de littérature jeunesse sont fréquents dans notre corpus. Ces images font référence pour la plupart à des héros issus de l'heroic fantasy, à des personnages de mangas ou de bandes dessinées (23 profils). La promotion de la littérature jeunesse afin de développer le goût et le plaisir de la lecture des élèves constitue une mission importante de cette communauté.

\section{Ethos d'expert numérique}

métier de professeur documentaliste et la fonction de veille informationnelle sont associés aux représentations (8) autour du domaine du numérique (arobase, QR code, nuage de tags, ordinateur). Le numérique prend une place importante dans le travail quotidien des professeurs documentalistes. Ces derniers doivent gérer les ressources numériques de l'établissement scolaire, assurer des séances pédagogiques associées à des enjeux de culture numérique et sensibiliser les enseignants à l'usage des technologies de l'information et de communication pour l'enseignement (TICE).

\section{Ethos d'enseignant}

La fonction enseignante, double composante de l'identité du professeur documentaliste, est affichée à travers des images indicielles (5) de l'école (tableaux, élèves, classe). Ce professeur assure une formation des élèves à la maîtrise et à la culture de l'information et travaille en collaboration avec les équipes pédagogiques.

\section{Ethos de médiateur culturel}

Le professeur documentaliste joue un rôle de passeur de culture dans l'établissement scolaire en tant que médiateur culturel. Les références (5) à des thèmes en lien avec l'histoire des arts (danse, musique, peinture, etc.) symbolisent cet investissement.

17 Les ethos identifiés (" professionnel du livre », « expert du numérique », « enseignant » et « médiateur culturel») composent les éléments d'une identité professionnelle collective. Ces ethos révèlent des proximités avec les compétences attendues par l'institution scolaire $^{4}$. Ce professeur est considéré comme le maître d'œuvre de l'acquisition par les élèves d'une culture de l'information et des médias (ethos d'«enseignant»), de l'organisation des ressources pédagogiques de l'établissement et de leur mise à disposition (ethos de "professionnel du livre» et d'«expert numérique »). Il est également acteur de l'ouverture de l'établissement sur son environnement éducatif, culturel et professionnel (ethos de «médiateur culturel»). L'étude des bannières juxtaposées aux comptes nous permet d'interroger un autre ethos, celui de " gestionnaire de l'espace documentaire", lié à la particularité du lieu d'exercice du professeur 
documentaliste. Plus spécifiquement, l'ethos professionnel est ici symbolisé par des photographies de CDI d'établissement scolaire. Cet ethos lié à l'espace documentaire associe intrinsèquement la fonction de professeur documentaliste au lieu de son activité, le CDI. La contribution de l'espace documentaire à la définition du métier est soulignée par Isabelle Fabre (2011) qui considère que le professeur documentaliste est à la fois un enseignant de la discipline information-documentation et le gestionnaire d'un système d'information pédagogique matérialisé dans le CDI. L'espace établit le cadre dans lequel se déroule l'action, il contribue à la formation de l'identité.

Parmi les représentations graphiques attachées aux bannières, nous distinguons la figure de l'espace paysage qui est spécialement adaptée à ce format en longueur. Nous retrouvons le thème de la nature présent dans certains avatars (24 productions imagées). Ces photographies s'avérent peu lisibles dans le cadre d'un avatar de dimension réduite. Ainsi, plusieurs images analysées mettent en scène la mer et l'horizon, la campagne, des fleurs et des arbres. Cette bannière allongée compose un système sémiotique similaire à celui présent dans les interfaces de blogs. Le blogueur a la possibilité d'intégrer une image personnelle ou d'en choisir une parmi une bibliothèque de formes. L'avatar apparaît au premier plan en superposition, la bannière constituant le second plan. La contrainte est d'y associer un thème qui permette tout de même une lisibilité du texte inscrit en blanc sur la bannière. La difficulté évoquée est sans doute la raison pour laquelle seulement $46 \%$ des comptes explorés proposent une bannière. Le caractère facultatif des bannières leur donne une valeur distinctive d'ordre esthétique. Ainsi, le dispositif structure la représentation graphique de l'identité.

En interagissant avec le dispositif, le sujet s'informe dans la structure prédéterminée par l'interface. L'emprise culturelle consiste, en somme, en les règles du jeu social présentées implicitement par la structuration de l'identité numérique. (Georges 2009 : 78)

Les choix révélés par les avatars des profils nous interrogent sur les lignes de continuité entre le réel et la représentation. Par conséquent, la représentation à l'écran de l'utilisateur ne fonctionne pas uniquement comme un outil d'identification, permettant ou non de le reconnaître hors ligne, mais avant tout comme un outil de positionnement et de reconnaissance au sein de la communauté en ligne dans laquelle il est actif. «Le processus de sémiotisation d'une représentation graphique en image de soi implique une habituation [du corps à interagir avec le dispositif], dans le flux de l'activité, de cette structure porteuse de valeurs symboliques» (Georges 2009: 78). L'ensemble des rituels sociaux de la communication qui sont déterminés par le dispositif, interviennent dans la définition de l'ethos collectif. Ce dernier n'est appréhendable qu'au travers des comportements individuels dans lesquels il vient s'incarner. Les individus, en se conformant à certaines normes en vigueur dans leur communauté, confirment les valeurs partagées du groupe professionnel, en attestant leur identification à ces valeurs collectives.

\section{L'audience dans la communauté de pairs}

Pour exister dans sa communauté, il est nécessaire de se manifester en ligne pour maintenir sa représentation de soi. Ainsi, la régularité des publications est un enjeu majeur pour accroître sa visibilité en ligne. Le Web 2.0 favorise l'identité agissante (produit de l'activité immédiate) et l'identité calculée (chiffrée) fondée sur les 
comportements informationnels. Pour exister dans sa communauté, il est nécessaire de se manifester en ligne pour maintenir sa représentation de soi. Les formes de l'identité agissante et calculée sont influencées par les possibilités offertes par le dispositif technosémiopragmatique.

L'économie d'un dispositif, son fonctionnement, déterminée par les intentions, s'appuie sur l'organisation structurée de moyens matériels, technologiques, symboliques et relationnels qui modélisent, à partir de leurs caractéristiques propres, les comportements et les conduites sociales (affectives et relationnelles), cognitives, communicatives des sujets. (Peraya 1999 : 153)

Plusieurs types de données chiffrées sont présents dans le profil public et nous informent sur l'activité du compte. À chaque profil sont associés des indicateurs chiffrés d'activité individuelle : le nombre de tweets produits, les abonnements à d'autres comptes et les abonnés au compte. La plateforme de micro-blogging Twitter est caractérisée par l'asymétrie des relations. En effet, la réciprocité n'est pas nécessaire entre les personnes suivies (following) et les personnes qui suivent les comptes: les abonnés (followers). Les relations sont établies sous la forme d'abonnements (followers/following). Pour la suite de notre analyse, nous nous appuierons sur des données chiffrées d'activité collectées en avril 2014.

\section{Les activités de la communauté}

Les actions et stratégies en ligne définissent à certains égards en creux la place de l'individu dans la communauté. La sociabilité professionnelle renvoie en outre à la manière dont il s'approprie les réseaux sociaux et dont il tisse des liens sur ce site de micro-blogging pour obtenir de l'information, échanger avec autrui ou encore se faire connaître. L'identité agissante correspond aux traces d'activité d'un utilisateur de Twitter. Elles sont issues de son interaction avec l'application. Ainsi, les abonnements choisis informent sur les relations interpersonnelles et les centres d'intérêt du sujet. Ces abonnements peuvent être complétés par le suivi de listes spécifiques. De la sorte, il est possible de regrouper selon un critère choisi des utilisateurs sous forme de liste. Lors de la sélection d'une liste, seuls les tweets des utilisateurs appartenant à celle-ci sont affichés. Les listes Twitter permettent de créer de cette manière des dossiers thématiques de comptes à suivre et de sources d'information incontournables dans un domaine d'expertise. Cette fonctionnalité est utilisée par $68 \%$ des internautes de notre corpus. De ce fait, cette possibilité proposée par la plateforme paraît tout à fait intéressante pour un professionnel de l'information habitué à catégoriser des sources. Par ailleurs, les utilisateurs de Twitter élaborent des listes personnelles pour organiser leur veille sur certains domaines comme dans l'exemple ci-dessous. 
Fig. 3. Les listes du compte de "Dora »

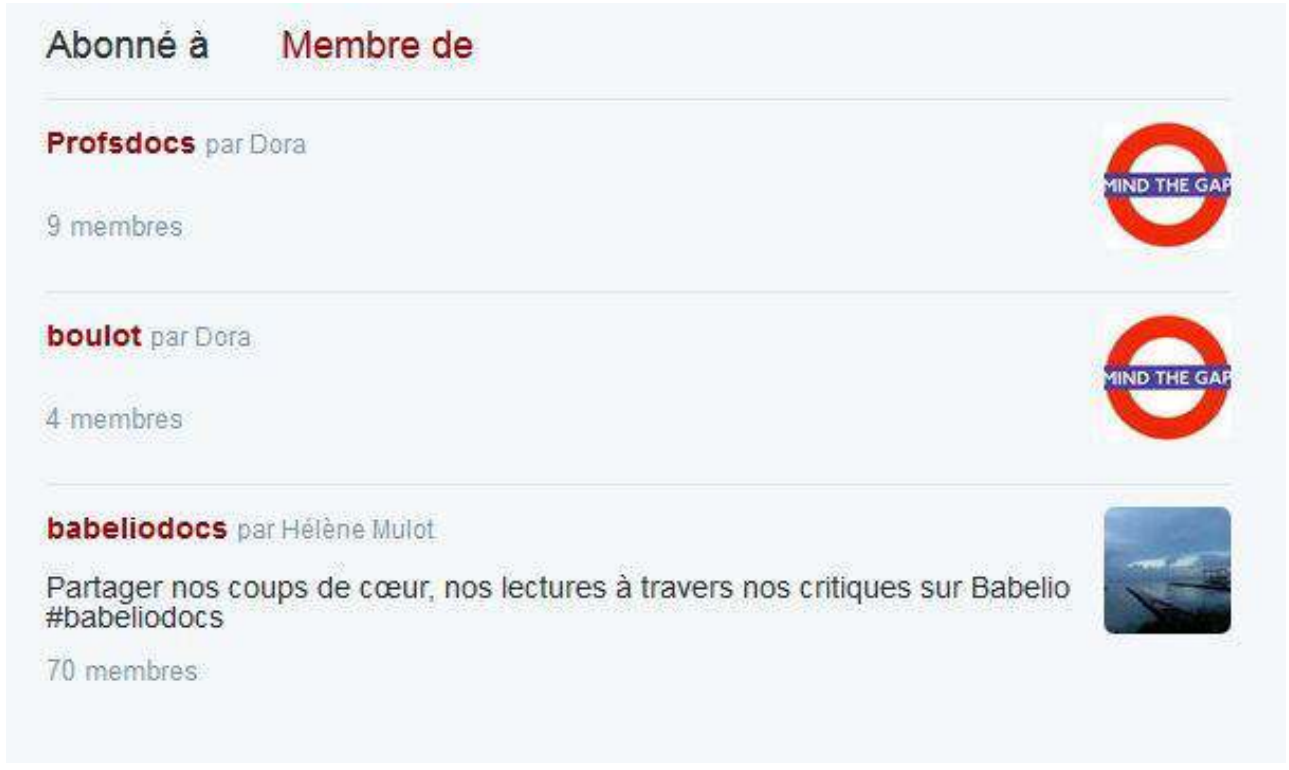
des sources d'information de nature pédagogique dans leur domaine de spécialité. Dans notre corpus, l'association aux profils de l'avatar par défaut (l'icône « œuf » est attachée à 14 profils) différencie les « observateurs ». Ces profils se révèlent par la suite peu actifs. Les pratiques généralisées de tests correspondent à la phase d'appropriation du dispositif. Les données étudiées sous l'angle de l'identité agissante nous permettent d'identifier les comptes actifs le temps d'une formation. L'utilisation comme indicateur d'activité du nombre global de messages envoyés représente une valeur absolue qui nous donne peu de renseignements sur l'activité momentanée d'un twitteur. Il apparaît plus approprié de rapporter cet indicateur sur le temps écoulé depuis la création du compte, voire de séquencer l'activité sur une échelle de temps prédéfinie. À l'instar de Fanny Georges (2008) lors d'une étude sur Facebook, nous avons évalué le "taux de présence » des différents profils explorés sur Twitter sur un temps limité. Ce taux correspond pour nous au nombre de jours nécessaire pour envoyer dix tweets. Cette information ne fait pas directement partie des métriques proposées par l'interface, elle permet cependant de quantifier la fréquence des actions de l'utilisateur sur Twitter. Le " taux de présence » manifeste l'identité agissante. Le groupe étudié met en moyenne 33 jours pour envoyer dix tweets. Le pourcentage de personnes dépassant cette durée moyenne pour envoyer 
dix tweets est de $23 \%$. Les membres de ce dernier groupe que nous qualifierons de «consommateurs passifs» ou «lurkers» (Bishop 2009) peuvent rester plusieurs mois sans avoir d'activité de production. A contrario, le taux de contributeurs qui produisent au moins dix tweets en une semaine est de $34 \%$. Dans une moindre mesure par rapport aux résultats observés sur des forums de discussion, nous retrouvons une participation inégale dans cette communauté en ligne. Les proactifs ne dépassent pas deux jours pour envoyer dix tweets ( $16 \%$ des profils). Cette « loi » de fréquence suit approximativement une distribution connue sous l'expression «loi de Pareto", qui correspond schématiquement au fait qu'un petit nombre de personnes contribue beaucoup alors que la plupart contribue peu, voire pas du tout aux échanges en ligne.

\section{Une nouvelle forme d'autorité}

Le premier indicateur de la visibilité d'un compte est le nombre de tweets publiés. Cet indice est le premier affiché sur un profil. Le nombre d'abonnés, c'est le «public imaginé » évoqué par Alice Marvick et Danah Boyd (2010), qui constitue une audience collective imaginaire. L'identité calculée se compose des variables quantitatives produites par le dispositif. De cette façon, le nombre d'abonnés est calculé automatiquement par le système à chaque nouvel abonnement ou désabonnement. Le nombre d'abonnés apparaît de prime abord comme un indicateur pertinent d'activité sur Twitter. Cependant, cette donnée chiffrée est confrontée à certaines limites, en particulier la pratique du massfollowing, qui consiste à suivre d'autres comptes en masse dans l'objectif qu'ils s'abonnent en retour (follow back). Nous avons appliqué à notre échantillon de profils de professeurs documentalistes des mesures pour évaluer l'activité globale sur Twitter. Le réseau social d'un compte est analysé à partir des différentes données chiffrées proposées.

Tableau 1 : Statistiques générales sur l'échantillon

\begin{tabular}{|c|c|}
\hline Profils de l'échantillon & 113 \\
\hline $\begin{array}{c}\text { Relations d'abonnement } \\
\text { Fréquence moyenne }\end{array}$ & $\begin{array}{c}50698 \\
448\end{array}$ \\
\hline $\begin{array}{c}\text { Relations d'abonné } \\
\text { Fréquence moyenne }\end{array}$ & $\begin{array}{c}59072 \\
522\end{array}$ \\
\hline
\end{tabular}

Nous constatons que le réseau social d'abonnés (tableau 1) est globalement plus dense (fréquence moyenne de 522) comparé au réseau d'abonnement (fréquence moyenne de 448). Pour la plupart des profils étudiés, l'audience de leur compte apparaît supérieure à leurs abonnements $(49 \%)$. La notoriété sur Twitter se mesure dans l'écart entre le nombre d'abonnements et d'abonnés, et ce différentiel est significatif au sein de la communauté des professeurs documentalistes. Nous avons essayé d'évaluer la mesure d'influence des différents utilisateurs de notre étude en appliquant un ratio followers / following (suiveurs / suivis). Le ratio Twitter se calcule en divisant le nombre des followers par le nombre de following. Il peut être positif - le nombre de followers est alors supérieur au nombre de following - ou bien négatif - le nombre de personnes suivies 
est supérieur au nombre de followers. Nous avons distingué trois degrés de ratio d'audience.

Tableau 2 : Résultats ratio d'audience

\begin{tabular}{|c|c|}
\hline Ratio d'audience & Résultats de l'échantillon \\
\hline $0<1$ & $40 \%$ \\
\hline $1<2$ & $41 \%$ \\
\hline $2>$ & $19 \%$ \\
\hline
\end{tabular}

Ratio inférieur à 1 : nouveaux twitteurs peu actifs, testeurs en phase d'exploration

Ratio de 1 à 2 : respect professionnel dans la communauté de pairs

Ratio de 2 à 5 : leader dans la communauté professionnelle, commentaires appréciés

Ces valeurs sont à modérer pour les comptes peu actifs, le calcul du ratio d'audience se révélant notablement élevé lorsque le compte affiche peu d'abonnement et beaucoup d'abonnés. Nous trouvons un relatif équilibre entre les comptes avec un ratio inférieur à 1 , révélateur d'une audience en construction et ceux entre 1 et 2 qui démontrent une régularité des échanges. Parmi les personnes avec un ratio supérieur à 2 , vingt comptes ${ }^{6}$ sont particulièrement actifs (nombre de tweets produits) et présentent davantage d'abonnements et d'abonnés que la moyenne de l'ensemble du corpus. Ces individus sont investis dans un processus de perfectionnement professionnel marqué par une démarche d'écriture réflexive sur leurs pratiques à travers leurs blogs. Pour certains, l'investissement dans leurs carrières les a conduit à changer de fonction. Ainsi, depuis le recueil de données, quatre professeurs documentalistes sont devenus respectivement bibliothécaire, chef d'établissement, ingénieur formation et chargé de mission numérique au Rectorat. Ils ont choisi pour la plupart un rôle de management dans l'institution scolaire, véritable changement du point de vue identitaire et synonyme d'un nouvel ethos professionnel.

Plusieurs attributs identitaires susceptibles d'influer sur les formes d'autorité informationnelle sont mis en évidence par Camille Alloing $(2014)^{7}$ lors d'une étude d'une sélection de profils Twitter. De cette façon, il identifie comme "expert» les profils qui regroupent un plus grand volume d'abonnés que d'abonnements. Le fait que les professeurs documentalistes soient suivis par un nombre important de personnes et que, parallèlement, ils suivent peu de comptes, démontre une certaine reconnaissance de leurs pairs en termes d'expertise sur leurs thématiques de prédilections. Camille Alloing caractérise les profils qui présentent un plus grand nombre d'abonnements que d'abonnés ( $34 \%$ de notre corpus) comme des «veilleurs mosaïques». Un faible volume d'abonnés peut questionner quant à leur légitimité ou la reconnaissance que leur portent les autres membres. Ces individus ont une propension à identifier de nouvelles sources et à effectuer un grand nombre de retweets (messages ayant fait l'objet d'une duplication par d'autres utilisateurs) sur des thématiques larges. Certains des profils étudiés qui affichent un différentiel négatif entre abonnements et abonnés se révèlent créés récemment ou peu actifs ( 11 profils). Cette catégorie d'« observateurs» est constituée d'entrants dans le métier et de «testeurs » de Twitter comme plateforme de veille et d'échange d'information. Ces comptes n'arrivent pas encore à atteindre un capital de 
visibilité suffisant dans la communauté. Au moment de la collecte des données, plusieurs comptes affichent une quasi-égalité entre abonnements et abonnés (17\%). Le dispositif sociotechnique de Twitter produit, de par sa performativité, une sociabilité et une autorité cumulative au fil du temps. L'observation des comptes un an plus tard montre une forte augmentation de toutes les métriques (volume et taux de croissance des abonnés).

Ce calcul d'audience nous informe sur la cohérence du groupe constitué et sur les interactions équilibrées entre les fonctions de partage et de recommandation. L'influence des professeurs documentalistes se construit quand ils se spécialisent, c'est-à-dire en postant régulièrement des messages sur un même sujet. Sur Twitter, les logiques de circulation d'information et d'influence ne se recouvrent pas nécessairement. Ainsi, Meeyoung Cha et al. (2010) ont montré que les différentes métriques d'influence sont indépendantes. Les auteurs ont comparé pour des utilisateurs, leur nombre de followers, de retweets et de mentions (citations par les autres utilisateurs). Ils ont observé que le nombre de followers n'était pas déterminé par le nombre de retweets et de mentions. Le nombre de followers est aussi lié à la place de l'individu dans une institution, à son statut (formateur Espé, intervenants Canopé, interlocuteur Tice ${ }^{8} .$. ) et à l'expertise qui lui est de fait reconnue. Les relations d'influence sont contextuelles et apparaissent liées à des groupes constitués.

\section{Conclusion}

L'étude des profils de professeurs documentalistes sur Twitter nous montre qu'ils participent activement à travers ce réseau social à la production de leur identité numérique en prolongement de leur identité officielle. L'articulation étroite qui existe entre la manière dont ils constituent différents traits de leur identité et la visibilité que leur accorde la plateforme compose un «design de la visibilité » (Cardon 2008) qui se fonde sur la mise en avant par Twitter de certains attributs / traces en interaction avec les tactiques des utilisateurs. La visibilité est un enjeu professionnel qui nécessite une appropriation des codes techniques et sociaux du dispositif et la mise en œuvre de stratégies de présence en ligne. De cette manière, les hashtags attribués par les professeurs documentalistes, internautes experts (Thiault 2015), correspondent à la prise en compte de l'usager comme récepteur. Ces traces numériques communes à un groupe d'individus se présentent comme des indices de l'expression d'une identité professionnelle, elles documentent les usages observés. La plateforme Twitter permet la mise en relation d'individus partageant des " univers communs » ou " ambient affiliation » (Zappavigna 2012). Les discours mis en relation forment un graphe d'univers partagés dans cette communauté professionnelle en ligne. Ce groupe s'intéresse particulièrement à l'innovation pédagogique portée par le numérique éducatif. La mutualisation des ressources apparait également au cœur de leur pratique professionnelle. L'information disponible pour tous dans un objectif de partage et de collaboration est source de savoir par la mise en commun des connaissances publiées. L'identité agissante comme somme des traces numériques que l'individu produit devient ainsi un bien commun pour la communauté.

31 À l'instar des journalistes qui affichent leur média de rattachement dans la twittosphère (Jouet 2015), les comptes des professeurs documentalistes sont significatifs d'une socialisation professionnelle marquée par la référence à l'établissement d'exercice (lieu et 
type d'établissement scolaire). Arnaud Mercier et Nathalie Pignard-Cheynel (2012) ont constaté que l'utilisation professionnelle de Twitter par les journalistes visait majoritairement à favoriser l'entre-soi, par le fait de suivre « ce que font les confrères, ce que publient les autres médias» (2012: 3). Dans notre enquête, les cinq ethos identifiés (professionnel du livre, expert du numérique, enseignant, médiateur culturel et gestionnaire de l'espace documentaire), révèlent une identité professionnelle collective tournée vers d'autres communautés proches. L'objectif sous-jacent est d'enrichir une professionnalité toujours en structuration par des emprunts à d'autres milieux professionnels que celui de l'école stricto sensu. Pour vérifier ce point, une analyse des comptes suivis par chaque profil nous éclairerait sur l'exogamie de la communauté. Il serait également nécessaire de mener des entretiens compréhensifs avec les membres les plus actifs de l'échantillon afin d'appréhender les processus d'élaboration individuels de l'ethos dans le contexte d'interactions en ligne.

\section{BIBLIOGRAPHIE}

Allard, Laurence et Blondeau, Olivier (dir.), 2007, Médiamorphoses, $\mathrm{n}^{\circ} 21$, « 2.0 ? Culture numérique, cultures expressives.

Alloing, Camille, 2014, « Vers une approche instrumentale de l'identité numérique : les attributs identitaires comme structuration de l'environnement informationnel ? », dans J.-P. Pinte (dir.), Enseignement, préservation et diffusion des identités numériques, Paris, Hermes Lavoisier, p. 39-68.

Bishop, Jonathan, 2009, « Increasing membership in online communities: The five principles of managing virtual club economies ", dans Proceedings of the Third International Conference on Internet Technologies and Applications (ITA 09), Wrexham, University of Wales Press, p. 12-20.

Broudoux, Évelyne, 2007, « Construction de l'autorité informationnelle sur le web », dans R. Skare, N. W. Lund et A. Varheim (dir.), A Document (Re)turn: Contributions from a Research Field in Transition, Francfort, Peter Lang, p. 1-11.

Cardon, Dominique, 2008, « Le design de la visibilité : un essai de cartographie du web 2.0. ", Réseaux, n 152, p. 165-193.

Casilli, Antonio, 2012, « Être présent en ligne : culture et structure des réseaux sociaux d'Internet ", Idées économiques et sociales, $\mathrm{n}^{\circ} 169$, p. 16-29.

Cha, Meeyoung, Haddadi, Hamed, Benevenuto, Fabricio et Gummadi, Krishna P., 2010, " Measuring user influence in Twitter: the million follower fallacy », ICWSM'10, AAAI, [En ligne], http://www.aaai.org/ocs/index.php/ICWSM/ICWSM10/paper/viewFile/1538/1826, 2011, consulté le $1^{\mathrm{er}}$ mars 2016.

Coutant, Alexandre et Stenger, Thomas, 2010, « Processus identitaire et ordre de l'interaction sur les réseaux socionumériques ", Les Enjeux de l'information et de la communication, nº 1, p. 45-64.

Fabre, Isabelle, 2011, « La contribution de l'espace documentaire à la définition d'un métier double : professeur-documentaliste », dans I. Fabre (dir.), Professeur-documentaliste, un tiers métier, Dijon, Educagri éditions, p. 191-208. 
Foucault, Michel, 1988, « Les techniques de soi », cours à l'université du Vermont, octobre 1982, dans Technologies of the Self, The University of Massachusetts Press, p. 16-49.

Georges, Fanny, 2008, « Les composantes de l'identité dans le web 2.0, une étude sémiotique et statistique. Hypostase de l'immédiateté ", Communication au $76^{\mathrm{e}}$ congrès de l'ACFAS, Web participatif: mutation de la communication, Québec, 6-7 mai, Institut national de la recherche scientifique.

Georges, Fanny, 2009, « Représentation de soi et identité numérique. Une approche sémiotique et quantitative de l'emprise culturelle du web $2.0 »$, Réseaux, nº 154, p. 165-193.

Jeanne-Perrier, Valérie, 2013, « Entre gestes sémiotiques et geste professionnelle : Twitter », dans A. Lévrier et A. Wrona (dir.), Matière et esprit du journal. Du Mercure galant à Twitter, Paris, PUPS, p. 263-277.

Jouët, Josiane et Rieffel, Rémy, 2015, «La sociabilité des journalistes sur Twitter. Entre performativité, ambivalence et multidimensionnalité ", Sur le journalisme, vol. 4, n 1 , [EN ligne], http://surlejournalisme.com/rev/index.php/slj/article/download/196/82, consulté le 10 mars 2016.

Marwick, Alice E. et Boyd, Danah, 2010, « I tweet honestly, I tweet passionately: Twitter users, context collapse, and the imagined audience », New Media \& Society, p. 114-133.

Mercier, Arnaud et Pignard-Cheynel, Nathalie, 2012, « Enquête sur les usages des réseaux sociaux par les journalistes français », OBSWEB, Université de Lorraine, [En ligne], http://obsweb.net/ blog/2012/05/14/enquete-sur-les-usages-des-reseaux-sociaux-par-les-journalistes-francais/, consulté le 10 mars 2016.

Peraya, Daniel, 1999, « Médiation et médiatisation : le campus virtuel », Hermès, nº 25, p. 153-168.

Rieder, Bernhard, 2010, « De la communauté à l'écume : quels concepts de sociabilité pour le “web social” ?", tic\&société, vol. 4, n 1, [En ligne], http://ticetsociete.revues.org/822, consulté le $1^{\mathrm{er}}$ mars 2016.

Thiault, Florence, 2015, « Le produsage des hashstags sur Twitter, une pratique affiliative », Questions de communication, $\mathrm{n}^{\circ}$ 28, p. 65-79.

Thiault, Florence, 2016, « Présentation de soi et promotion de l'identité professionnelle sur Twitter », dans A. Kiyindou, F. Barbey et L. Corroy-Labardens (dir.), De l'éducation par les médias à l'éducation aux médias, Paris, L'Harmattan, p. 53-64.

Weber, Max, [1905] 1996, L'Éthique protestante et l'Esprit du capitalisme, Paris, Plon.

Zappavigna, Michele, 2012, Discourse of Twitter and Social Media, Londres, Bloomsbury Academic.

Zarca, Bernard, 2009, « L'ethos professionnel des mathématiciens », Revue française de sociologie, vol. 50, p. 351-384.

\section{NOTES}

1. "L'enquête sur les pratiques informationnelles, 2ème édition", Savoirs Cdi, https:// www.reseau-canope.fr/savoirscdi/metier/diversite-du-metier-de-professeur-documentaliste/ enquete-sur-les-pratiques-informationnelles-2eme-edition.html, mai 2014.

2. Nous avons considéré les comptes ayant produit moins de 50 tweets entre 2011 et 2014 comme inactifs. 
3. Les pseudonymes et avatars présentés dans les exemples 1 et 2 ont été choisis en raison de leur prototypie ; leur valeur est illustrative plus que représentative.

4. Référentiel des compétences professionnelles et des métiers du professorat et de l'éducation. Compétences spécifiques aux professeurs documentalistes (Bulletin Officiel, $\mathrm{n}^{\circ} 30,25$ juillet 2013).

5. Babelio est un réseau social destiné à enregistrer des bibliothèques personnelles qui peuvent ensuite être partagées et commentées par les autres utilisateurs.

6. Ces comptes présentent quelques caractéristiques spécifiques: ancienneté de l'adhésion à Twitter (2009-2010), liens pour la moitié d'entre eux vers des blogs personnels (de littérature jeunesse ou pédagogique), changement de fonction pour quatre professeurs documentalistes.

7. Recherche action en entreprise à La Poste Courrier.

8. Dans chaque académie, un interlocuteur TICE et Documentation, désigné par le Recteur en liaison avec le DAN (Délégué académique numérique) et l'inspection pédagogique régionale, participe aux réunions de coordination nationales, aux échanges et aux travaux interacadémiques mis en place dans le cadre de ce réseau.

\section{RÉSUMÉS}

L'article présente une recherche sur les fonctions du réseau social numérique Twitter dans l'ethos d'une communauté professionnelle identifiée. Cette étude exploratoire s'appuie sur un corpus de profils constitué sur le critère de l'affiliation à la communauté professionnelle des professeurs documentalistes. Le réseau social numérique Twitter est le lieu de stratégies de la part des acteurs, au service d'une mise en visibilité dans le cadre d'une socialisation et d'un engagement professionnel. Le modèle sémiotique de l'identité numérique est appliqué pour identifier les différents ethos numériques de ces professionnels.

The article presents the findings of a study on the use of a digital social network Twitter in ethos of a specific professional community. This study is based on a corpus of profils from users belonging to the community of school librarian teachers. The digital social network Twitter has become a strategic tool for users, whose socialization and professional commitment. The semiotic model of digital identity is applied to identify the various digital ethos of these professionals.

\section{INDEX}

Keywords : ethos, school librarian, social network sites, professional identity

Mots-clés : professeur documentaliste, Twitter, réseaux sociaux numériques, identité professionnelle

\section{AUTEUR}

\section{FLORENCE THIAULT}

Université de Lille 3, laboratoire Gériico (EA 4073) 